\title{
The hydrological aspects of sustainable development
}

\author{
R. Vedom \\ Hydrology and Environment, Canada
}

\begin{abstract}
The purpose of this article is to introduce the Harmonized Frequencies Analysis $^{\mathrm{TM}}$ (HFA), the method that considers the water cycle dynamics at various scales, and to investigate its ability to assess the watershed development process in order to specify the hydrological criteria for its sustainability. The method is based on the Law of the Structural Stability of Systems and considers river flow as an intrinsic part of the entire water dynamic system which is responsible for temperature control.

The Humber River watershed, the lower part of which is highly urbanized and contaminated, was assessed for sustainability at hourly, daily, monthly, and yearly scales for the period of 1953-2006.

The quantitative increase of the base and inter components of river flow caused by the conventionally proper storm water management in combination with the increasing contamination and lack of vegetation resulted in alteration of the water cycle's functional profile, i.e. the drainage function took over the temperature control. This is characterised by the increasing trend of instability at all scales, pulling out tornado as the mechanism of self-stabilization at the hourly scale.

Based on the analysis results, we concluded that HFA is the reliable tool for the watershed sustainability assessment, which is defined as the functional stability of the hydrological cycle within a developing spacetime.
\end{abstract}

Keywords: indivisibility of hydrosphere, dynamic structure of hydrosphere, Harmonized Frequencies Analysis, sustainable development. 


\section{Introduction}

The purpose of this article is to specify the hydrological criteria for a local water resources sustainable development using the Harmonized Frequency AnalysisTM (FHA), a new hydrological method considering the indivisibility and functionality of hydrosphere and the water cycle. The need for such extended consideration of the local water resources is based on the increasing dynamic instability of the global water resources which manifests itself in the ocean level rise, flooding, tsunami, tornado, disappearing of glaciers, water scarcity, etc.

Sustainable development, in the frame of Earth System prediction, has a "multiple-paradigm" definition which includes optimization, stabilization or pessimization scenario. It is due to the fact that sustainability, according to Murtugudde [1], is more intuitive than theoretical perception. Water resources and their management have been mostly addressed at a local to basin scale (Hoff [2]), at which a mathematical definition of sustainability would require the rate of the system change equal to zero (Murtugudde [1]). This only indicates the incorrect mathematical definition of the system sustainability as not being a dynamic one.

The dynamic structure of any natural system is the manifestation of the Law of Structural Stability of Systems formulated by the Byelorussian philosopher and mathematician Eduard Soroko in 1984 [3], which is based on the generalised principle of the Golden Section as the attractor. The law states the following: "The Generalised Golden Sections are invariants, which allow natural systems in process of their self-organisation to find harmonious structure, stationary regime of their existence, structural and functional stability" (Stakhov [4, p.252]). According to this law, hydrosphere and the water cycle is a natural system that could be defined as follows. The hydrological cycle is the driven by solar energy and the planetary movements continuous exchange of water gaseous, liquid, and solid phases within the indivisible hydrosphere (the collective dynamic mass of water found above, on and under the Earth's surface in atmosphere, criosphere, biosphere, rhizosphere, ground-, and surface water including the ocean). This exchange, keeping the life-sustaining temperature regime, creates a specific for time and space dynamic structure of the entire hydrosphere, which is a solid base for water, energy, and matter balances. Interdependence and interaction of these balances are conceptually well known as the substance cycles for carbon, nitrogen, sulfur, phosphorus, etc. The water cycle is known as the solvent, transport media, and driving force for them [5,6]. Due to Earth movements all these cycles have two main temporal scales: daily and yearly representing certain functional timeframe, in which the asymptotic stability of the yearly fluctuations is the limiting factor for all other scales. Each element of the indivisible hydrosphere has its own role and the corresponding dynamics, and is very interdependable within the overall function of temperature control. Atmosphere, the most dynamic at the instant scale, is responsible for transport of the precipitable atmospheric water around the globe toward the highest positive instant temperature gradients, which, generally, occur on the continental part of the globe increasing from equator toward the poles (Peixoto [8]). At the yearly scale 
the precipitable water is distributed in accordance with temperature and the corresponding saturation capacity of air. Actual daily precipitation characterizes the release of the daily tension created by temperature gradient. The instant gradients of temperature are the driving force for the atmospheric dynamics (Doswell and Bosart [17]). Criosphere, ocean and groundwater are water and energy storages with their own slow dynamics feeding the instant dynamic needs of atmosphere, therefore, their daily dynamics mostly occur in the relatively thin boundary layer, which terrestrial equivalent is surface and soil water, and are extremely interrelated with the atmospheric one. Biosphere, particularly vegetation, facilitates this multi-scale interaction, knowledge of which has been successfully used in water management (Xie et al. [13]). Other function of biosphere is correction of the atmosphere-surface-groundwater interface which includes: reflection (albedo), absorption, and storage capacity for solar energy of different elements of hydrosphere creating the biospheric buffers in different forms (Rowntree and Dumenil [9]). The terrestrial buffer (vegetation and rhizosphere), being a groundwater-atmosphere facilitator in the water cycle, regulates both the evapotranspiration and condensation processes via the area and size of its leaves, stems, and roots in accordance with the energy flux, quality of groundwater and air (Bouten [7]). In order to facilitate water and heat transformation from the terrestrial storages to atmosphere in all scales, biosphere dynamics occur in the same scales as well. The tension created during the day time by the light and heat fluxes at any point is released as the latent heat via condensation locally in atmosphere as clouds, fog, rain, snowfall, and nightly on the surface as due or frost. Precipitation is, therefore, a volumetric measure of tension released. It means that the certain gradient of daily precipitation corresponding to the released tension is bounded with the certain gradient of daily temperature. The gradients of all other variables in correspondence with the temperature and precipitation ones create the certain dynamic structure of the entire hydrosphere. Mathematicians call similar type of the deterministic finite dimensional system stability UBIBO: uniformly bounded-input bounded-output (Sontag [21]). The assessment of these components and the possibility to control them (as vegetation does) is the paramount task for scientists and practitioners. Understanding of the natural mechanism of both physical and biological bounding in the buffer provides the basis for sustainable management of the buffer capacity.

Returning to the Law of Structural Stability, it is necessary to emphasize that self-stabilization of any natural system is its aspiration to some invariance (attractor) in its dynamic structure composition. Therefore, the hydrological requirement for sustainable development is the continual control and support of this aspiration to functional and structural stability of the entire water cycle at any developing spacetime.

\section{Method}

Technically, HFA, being a hydrological tool for sustainability assessment, is a solution for the water cycle and hydrosphere indivisibility, functionality, and 
scale-invariance; mathematically it is a combination of asymptotic, uniformly bounded, and structural stability of the dynamic water system; statistically, it is manifestation of the "componential" independency of the system's variables.

\subsection{The Harmonized Frequencies Analysis ${ }^{\text {TM }}$}

Methodologically, HFA, based on the water cycle functionality and indivisibility, consists of three main components, two of which are tightly interrelated: 1) estimation of the water dynamic structure of any examined spacetime for all available scales using the SimpleBase Delineation Model ${ }^{\text {TM}}$; 2) its graphical visualisation as the Structural Harmony Chart of Hydrosphere ${ }^{\mathrm{TM}}$ (SHC) (Vedom [15, 16, 23]). The third component is the assessment of the entire structure stability and the impact of selected variables using indexation and cross-correlation techniques.

\subsubsection{Base component delineation}

The first step in the SimpleBase Delineation Model ${ }^{\mathrm{TM}}$ is conducted independently for each variable. The frequency function of the time-step gradients $d Q=f(N)$ in the frequency domain (Fig 1) is analysed in order to find the most frequent one, which corresponds to the gradient of the asymptotic controllability (stability) (Sontag [21]).

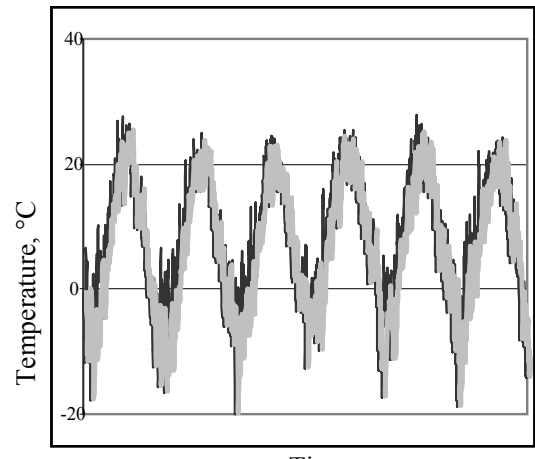

Time

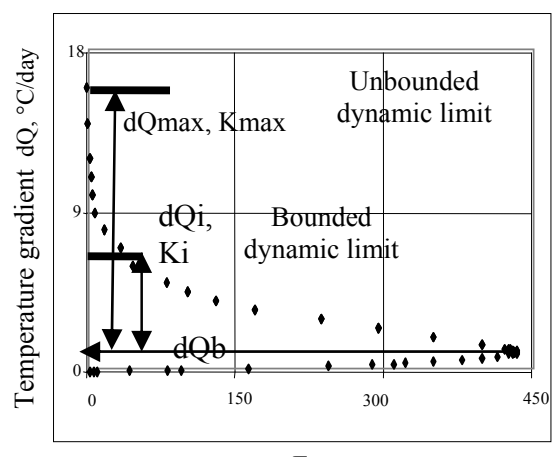

Frequency

Figure 1: Separation of daily temperature in the time (left) and frequency (right) domains. The grey line outlines the base component in the time domain.

The found gradient $\mathrm{dQb}$ outlines the base component $\mathrm{B}$ (the average base amplitude in decimals of the average total amplitude) of any variable in the time domain and is called, therefore, the base dynamic limit. There is no formula for any custom convolution digital filter (Smith [20]), but the algorithm can be expressed mathematically (Vedom [15]):

$$
\text { if } \mathrm{Q}_{\mathrm{t}}-\mathrm{Q}_{\mathrm{t}-1}>\mathrm{dQb}, \mathrm{Qb}_{\mathrm{t}}=\mathrm{Q}_{\mathrm{t}-1}+\mathrm{dQb} \text {, otherwise } \mathrm{Qb}_{\mathrm{t}}=\mathrm{Q}_{\mathrm{t}} \text {, }
$$


where $\mathrm{Q}_{\mathrm{t}}$ is the total value of a variable at the moment $\mathbf{t}$; $\mathrm{Q}_{\mathrm{t}-1}$ is its total value at the previous moment $\mathbf{t}-\mathbf{1} ; \mathrm{Qb}_{\mathrm{t}}$ is the base component of the variable at the moment t. Duration of the "moment" is equal to the series time-step of an examined scale: hour, day, month or year.

The end of a flux is determined as decrease or unchanged condition following an increase of the variable:

$$
\mathrm{Q}_{\mathrm{t}}=\mathrm{Qb}_{\mathrm{t}}, \mathrm{Q}_{\mathrm{t}-1}>\mathrm{Qb}_{\mathrm{t}-1}, \mathrm{Q}_{\mathrm{t}+1}>=\mathrm{Qb}_{\mathrm{t}+1}
$$

The opposite end of the frequency curve (Fig. 1) identifies the extreme timestep gradient of the variable dQmax corresponding to a single occurrence of this positive time-step change during the period.

\subsubsection{Inter and storm components separation and SHC creation}

The second step is separation of inter and storm gradients, which is conducted first at the daily scale due to its clear functional interpretation and use.

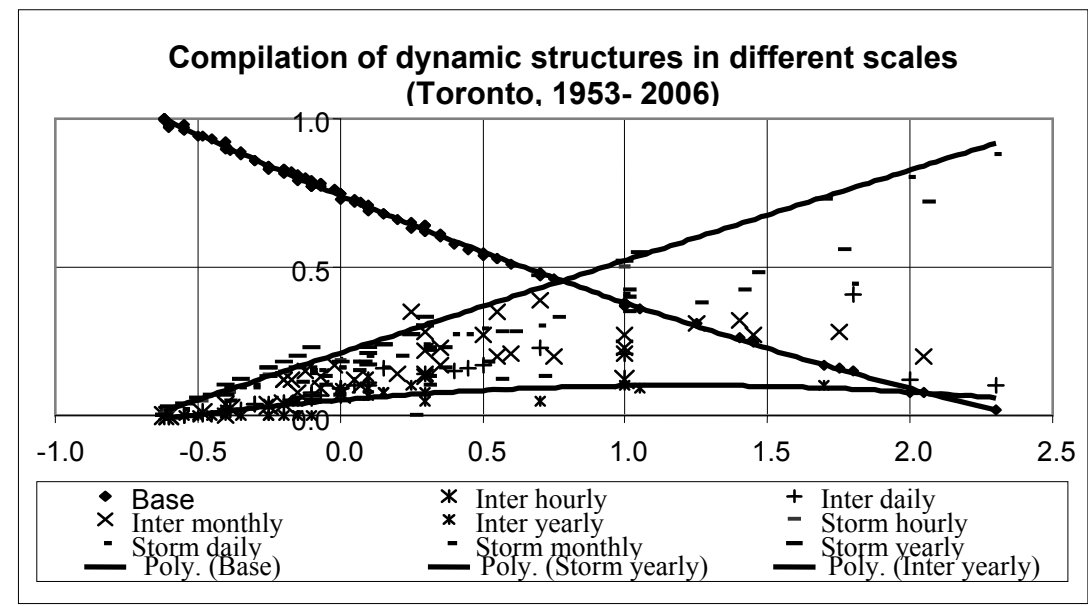

Figure 2: $\quad$ SHC of the Toronto-Humber R.-Lake Ontario-1953-2006 spacetime in four scales.

The visualisation of this procedure is shown as the Structural Harmony Chart of Hydrosphere ${ }^{\mathrm{TM}}$ (Fig. 2). Methodically, the synchronization process consists of the semi-empirical procedure to find the exact parameters of SHC for the examined spacetime. All variables are ranged according to their base component $B$ and are placed on the base curve $B=f(K i)$ between two certain and one spacetime-specific points controlled by the Law of Structural Harmony of System. $\mathrm{Ki}$ is the structural divider defining the ratio between the base and inter dynamic limits, dQb and dQi. Mathematically, the line between the extreme and inter dynamics, dQi, outlining the sum of inter and base components in the time domain (Fig 1), is defined by the following empirical equation:

$$
\mathrm{dQi}=\mathrm{dQb} * 2^{(\mathrm{Ki}+0.618)}
$$


where dQi is the inter or bounded dynamic limit of each variable; 0.618 is the Golden Section or Golden Ratio (Stakhov [4]). The structural divider Ki, defining the ratio between the base and inter dynamic limits, automatically defines the storm component as well as the difference between 1 and the sum of the base and inter components. The equation (3) is an empirical expression of the Law of Structural Harmony of Systems for hydrological systems, initially applied only for the stream flow (Vedom $[15,16])$. According to this equation, the first certain point of the $\mathrm{B}=\mathrm{f}(\mathrm{Ki})$ curve, named "the point of zero tension", has the following coordinates $\mathrm{Ki}=-0.618$ and $\mathrm{B}=1$. The second point, named "the equilibrium tension point", has the following coordinates $\mathrm{Ki}=1$ (making the sum in the brackets equal to 1.618 ), $\mathrm{B}=0.382$. This point divides the total amplitude of any variable having $\mathrm{Ki}=1$ "in extreme and middle ratio" (the Golden Section). Ideally, the rest of the gradient at this point is also divided between storm and inter components in the same ratio creating the following "golden" structure: $\mathrm{B}=\mathrm{S}=0.382, \mathrm{I}=1-2 * 0.382=0.236$. In reality, different scales of the same spacetime have different interrelated capacities to reach the harmony.

The third point of the base curve, developed at the daily scale, belongs to precipitation as the tension releasing variable in the temperature control process and has the site and time specific coordinates. In the model the resonance condition between precipitation and temperature means defining the value of $\mathrm{Ki}$ for the daily precipitation with preliminary estimated value of traces that provides the equal frequencies for the inter (bounded) dynamic limit of precipitation and the base (stable) dynamic limit of temperature. The defined value of $\mathrm{Ki}$ for precipitation corresponds to the utmost right margin of the graph (Fig. 2).

When the third point of the base curve $\mathrm{Ki}=\mathrm{f}(\mathrm{B})$ is defined, $\mathrm{Ki}$ values for all other variables are placed on the base curve $\mathrm{Ki}=\mathrm{f}(\mathrm{B})$ according to their base components with the least squares $\mathrm{Rb}^{2} \geq 0.999$. The bounded and unbounded constituents of all variables will be maintained according to their Ki values. At this point the daily scale SHC is completed.

\subsubsection{Hourly, monthly and yearly SHCs}

The base curve of SHC at each scale has the same parameters because it represents the same stable part of the same spacetime's dynamics estimated independently for each variable. The position of variables at the base curve, however, will be different for different scales, as well as the shape and parameters of the storm (unbounded) and inter (bounded) curves. Their interrelation depends on the values of $\mathrm{Ki}$ associated with a certain position of a variable at the base curve. The base curve has the same three basic points at any scale providing the benchmark for all examined variables at all scales (Fig. 2).

\subsubsection{Assessments}

The indivisible and scale-invariant dynamic structure is the basis for the parametrical assessments of the water system stability.

The lump criteria of the entire system stability are the yearly base $(\mathrm{dQb})_{\mathrm{y}}$ and inter (dQi) y dynamic limits for temperature: at any other scale the numerical base 
dynamic limit $\mathrm{dQb}$ for temperature should not be less than the base dynamic limit at the yearly scale: $(\mathrm{dQb})_{\mathrm{y}}<\mathrm{dQb}$.

Frequencies of variables $(\mathrm{Nb}, \mathrm{Ni})$ tell about the unconditionally stable and bounded periods of their fluctuations.

The structural position of a variable is characterised by the value of its structural divider $\mathrm{Ki}$ : if $\mathrm{Ki}<1$ (Fig. 2), the base component is generally bigger than the storm one; if $\mathrm{Ki}>1$, the storm component is bigger than the base one. The increasing trend of $\mathrm{Ki}$ characterises the destabilisation process with the growing storm component. If $\mathrm{Ki}$ of a variable is bigger than the $\mathrm{Ki}$ for precipitation, this variable creates an energy tension bigger than could be released on the daily basis.

Phases of storm-inter shares at different scales oscillating at the equilibrium point around their long-term averages characterise the temporal status of the spacetime stability (aspiration to the attractor) at each scale.

The Hydrosphere Elasticity Index (HEI), which is literally the stress-strain ratio of the unbounded component of a variable characterises the ability of hydrosphere to control the energy fluctuations through each variable:

$$
\mathrm{HEIi}=(\mathrm{Si}-\mathrm{Ii}) /(\mathrm{Bt}-\mathrm{It}) *(\mathrm{Ki}-\mathrm{Kt})
$$

where Si and Ii are the storm and inter shares of the i-variable; Bt and It are the base and inter shares of temperature; $\mathrm{Ki}$, and $\mathrm{Kt}$ are the structural dividers for the $\mathrm{i}$-variable and air temperature, respectively. HEI indicates the functional position of a variable in the dynamic structure: the higher HEI of a variable, the more unstable energy it gains within the examined spacetime. Precipitation has the highest positive value of HEI at daily scale, indicating the functional limit for energy consumption. If HEI of a variable is negative, it indicates its capacity for self-stabilization.

The power of base, inter and storm dynamics, PBD, PID, PSD, correspondingly, are the parameters characterising the power allocated in its different dynamic components of a variable:

$$
\begin{gathered}
\text { PBD }=\mathrm{dQb} * \mathrm{Nb} * \mathrm{HEI}, \\
\mathrm{PID}=\mathrm{dQ}{ }^{*} \mathrm{Ni}^{*} \mathrm{HEI}, \\
\mathrm{PSD}=\mathrm{dQmax}{ }^{*} \operatorname{Nmax}^{*} \mathrm{HEI}
\end{gathered}
$$

where dQb, dQi and dQmax are the base, inter and storm dynamic limits; $\mathrm{Nb}, \mathrm{Ni}$ and Nmax are frequencies of their occurrence during the time period. HEI in this case characterises positive or negative power, which corresponds to the external distribution or internal consumption for self-stabilization. Dimension of the power parameters is the dimension of the variable itself $-\mathrm{m}$ for the level, $\mathrm{mm}$ for precipitation, $\mathrm{m} / \mathrm{sec}$ for speed, etc. The power criteria for the entire system stability are not developed yet. 


\subsubsection{Cross-correlation analysis}

When development of SHC for the examined spacetime is completed, the crosscorrelation analysis can be conducted in order to find two of the most controversial variables or their components at each scale. One of them has the highest regular average correlation coefficient; the other has the highest average of their absolute values. There are only selected "not-related" variables in this cross-correlation, which is the standard requirement for any statistical analysis (Berthouex and Brown [10]). For this reason the totals of chosen variables and their components are analysed separately to "avoid" their inter-influence.

\section{Data}

The following data were considered for the same period: meteorological data at the Pearson Airport, the lower Humber River flow quantity and quality at Weston $\left(800 \mathrm{~km}^{2}\right.$ out of $903 \mathrm{~km}^{2}$ of the total watershed area), the level in the Lake Ontario at Toronto. The meteorological data (7 variables of 473352 hours: air temperature, dew point, relative humidity, air pressure, wind speed and direction, visibility) at the Pearson International Airport (1953-2006) were converted into the daily averages and extremes (21 variables of 19723 days). Then they were considered along with daily precipitation and snow cover at the same location, flow of the Humber River, and the level in the Lake Ontario (totally 25 variables of 19723 days each). Then all these variables were converted into the monthly and yearly averages and extremes (33 variables of 648 months and 54 years).

The increase of population and chlorides concentrations (Table 2) were considered as indicators of development in the Humber River watershed. Yearly population data was provided by the Peel Region Municipality. Daily chloride concentrations for the period of 1988-2006 were assessed based on the sampling results in accordance with the transport capacity of the separated flow (Vedom [14]). Pentad averages for the 1953-1987 periods were estimated by interpolation, accepting the initial concentration of chlorides as large as $10 \mathrm{mg} / \mathrm{l}$.

\section{Results and discussions}

The partial results of the lump set processing at four scales are shown in Table 1 and on Figure 2. The pentad results are presented in Table 2.

Hourly base dynamic limit $\mathrm{dQb}$ for temperature is numerically equal to the yearly bounded one, dQi, $0.49^{\circ}$ (Table 1 ). The pentad results indicate the decreasing trend of temperature stability at all scales: the values of Ki have the increasing trend, the hourly $\mathrm{dQb}$ has become equal to the yearly dQb $0.29^{\circ}$, since the late 70 s (Table 2). This indicates the marginal stability of the water system at the hourly scale. 


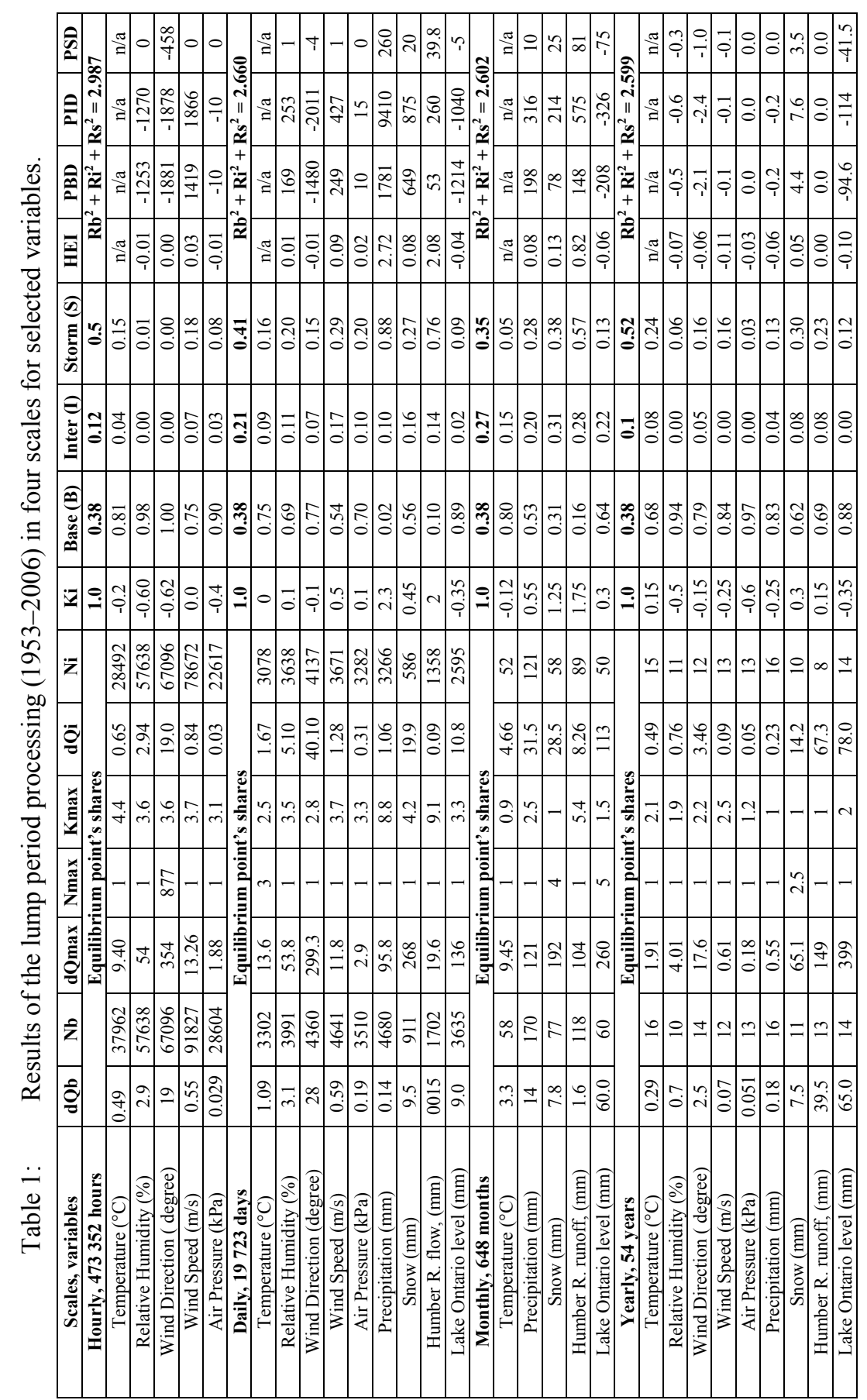


Table 2: $\quad$ Results of the dynamic structure analyses by pentads in three scales for selected variables and parameters.

\begin{tabular}{|c|c|c|c|c|c|c|c|c|c|c|c|}
\hline $\begin{array}{l}\text { Scales/ } \\
\text { variables, parameters }\end{array}$ & $\begin{array}{l}1953- \\
1957\end{array}$ & $\begin{array}{l}1957- \\
1961\end{array}$ & $\begin{array}{l}1962- \\
1966\end{array}$ & $\begin{array}{l}1967- \\
1971 \\
\end{array}$ & $\begin{array}{l}1972- \\
1976\end{array}$ & $\begin{array}{l}1977- \\
1981 \\
\end{array}$ & $\begin{array}{l}1982- \\
1986\end{array}$ & $\begin{array}{l}1987- \\
1991 \\
\end{array}$ & $\begin{array}{l}1992- \\
1996 \\
\end{array}$ & $\begin{array}{l}1997- \\
2001\end{array}$ & $\begin{array}{l}2002- \\
2006\end{array}$ \\
\hline \multicolumn{12}{|l|}{ Hourly } \\
\hline Temperature, $\mathrm{Ki}$ & -0.3 & -0.3 & -0.25 & -0.25 & -0.3 & -0.15 & -0.15 & -0.15 & -0.15 & -0.1 & -0.1 \\
\hline Temperature, dQb & 0.49 & 0.49 & 0.49 & 0.49 & 0.49 & 0.29 & 0.29 & 0.29 & 0.29 & 0.29 & 0.29 \\
\hline Relative humidity, dQb & 2.9 & 2.9 & 1.9 & 1.9 & 1.9 & 1.9 & 1.9 & 2.9 & 1.9 & 1.9 & 1.9 \\
\hline equilibrium point, I & 0.1 & 0.14 & 0.12 & 0.09 & 0.15 & 0.1 & 0.15 & 0.12 & 0.12 & 0.11 & 0.15 \\
\hline equilibrium point, $\mathrm{S}$ & 0.52 & 0.48 & 0.5 & 0.53 & 0.47 & 0.52 & 0.47 & 0.5 & 0.5 & 0.51 & 0.47 \\
\hline \multicolumn{12}{|l|}{ Daily } \\
\hline Temperature, $\mathrm{Ki}$ & -0.1 & -0.04 & 0.1 & -0.15 & -0.1 & -0.2 & -0.15 & -0.15 & 0 & -0.1 & 0.05 \\
\hline Relative humidity, dQb & 2.9 & 2.5 & 3.5 & 2.7 & 3.5 & 2.9 & 3.1 & 3.1 & 3.1 & 3.7 & 3.2 \\
\hline Relative humidity, dQmax & 33.2 & 37.8 & 37.4 & 33.2 & 34.9 & 31.0 & 33.1 & 43.7 & 40.8 & 34.4 & 45.1 \\
\hline Relative humidity, dQi & 5.1 & 4.7 & 5.6 & 4.7 & 5.6 & 4.6 & 5.0 & 5.3 & 5.5 & 6.1 & 5.6 \\
\hline Max Wind Direction, dQb & 19 & 29 & 19 & 19 & 19 & 19 & 19 & 19 & 23 & 25 & 29 \\
\hline Min Wind Direction, dQb & 19 & 19 & 17 & 17 & 9 & 7 & 7 & 7 & 7 & 7 & 9 \\
\hline Max Wind Speed, dQb & 0.77 & 0.79 & 0.77 & 1.04 & 0.77 & 0.87 & 0.99 & 1.04 & 1.05 & 1.04 & 1.35 \\
\hline Min Wind Speed, dQb & 0.47 & 0.47 & 0.47 & 0.47 & 0.57 & 0.45 & 0.57 & 0.57 & 0.57 & 0.57 & 0.57 \\
\hline Humber R., Base share B & 0.04 & 0.06 & 0.05 & 0.12 & 0.04 & 0.15 & 0.12 & 0.15 & 0.09 & 0.10 & 0.13 \\
\hline Humber R., Inter share I & 0.05 & 0.09 & 0.08 & 0.17 & 0.11 & 0.20 & 0.19 & 0.19 & 0.13 & 0.15 & 0.22 \\
\hline Humber R., Storm share S & 0.90 & 0.85 & 0.87 & 0.71 & 0.85 & 0.66 & 0.69 & 0.66 & 0.78 & 0.75 & 0.65 \\
\hline equilibrium point, I & 0.18 & 0.23 & 0.21 & 0.2 & 0.28 & 0.24 & 0.25 & 0.24 & 0.23 & 0.19 & 0.26 \\
\hline equilibrium point, $\mathrm{S}$ & 0.44 & 0.39 & 0.41 & 0.42 & 0.34 & 0.38 & 0.37 & 0.38 & 0.39 & 0.43 & 0.36 \\
\hline \multicolumn{12}{|l|}{ Monthly } \\
\hline Temperature, Ki & -0.6 & -0.55 & -0.1 & -0.6 & -0.55 & -0.5 & -0.5 & -0.5 & -0.5 & -0.35 & -0.15 \\
\hline equilibrium point, I & 0.2 & 0.22 & 0.2 & 0.24 & 0.23 & 0.25 & 0.24 & 0.2 & 0.24 & 0.21 & 0.3 \\
\hline equilibrium point, $\mathrm{S}$ & 0.42 & 0.4 & 0.42 & 0.38 & 0.39 & 0.37 & 0.38 & 0.42 & 0.38 & 0.41 & 0.32 \\
\hline \multicolumn{12}{|l|}{ Yearly averages } \\
\hline Temperature, ${ }^{\circ} \mathrm{C}$ & 8.1 & 7.8 & 7.3 & 7.0 & 7.2 & 7.1 & 7.6 & 8.4 & 7.5 & 9.2 & 8.8 \\
\hline Relative Humidity (\%) & 75.9 & 73.1 & 73.5 & 76.3 & 76.2 & 75.3 & 75.2 & 72.1 & 74.0 & 68.6 & 70.8 \\
\hline Wind Direction (degree) & 22.4 & 22.3 & 22.4 & 22.3 & 21.0 & 21.5 & 20.7 & 21.0 & 20.3 & 21.3 & 21.0 \\
\hline Wind Speed $(\mathrm{m} / \mathrm{s})$ & 4.6 & 4.6 & 4.2 & 4.1 & 4.3 & 4.0 & 3.9 & 4.1 & 3.9 & 4.2 & 4.4 \\
\hline Air Pressure $(\mathrm{kPa})$ & 99.5 & 99.5 & 99.4 & 99.4 & 99.4 & 99.5 & 99.6 & 99.5 & 99.6 & 99.5 & 99.5 \\
\hline Precipitation, $\mathrm{mm}$ & 767 & 657 & 735 & 754 & 824 & 835 & 850 & 704 & 845 & 686 & 789 \\
\hline Snow, $\mathrm{cm}$ & 4.3 & 5.5 & 6.3 & 7.9 & 7.3 & 6.2 & 6.8 & 4.1 & 3.6 & 4.1 & 8.8 \\
\hline Lake Ontario level, m & 75.0 & 74.6 & 74.5 & 74.8 & 75.0 & 74.8 & 74.9 & 74.8 & 74.8 & 74.8 & 74.8 \\
\hline Humber R. flow, m3/s & 7.1 & 4.3 & 3.8 & 5.6 & 6.3 & 6.7 & 8.2 & 5.4 & 6.9 & 5.8 & 7.3 \\
\hline Population, /1000 & 100 & 106 & 125 & 158 & 200 & 270 & 335 & 410 & 488 & 670 & 710 \\
\hline Urbanised area, $\%$ & 3.6 & 4.1 & 4.9 & 6.2 & 7.8 & 10.6 & 13.1 & 16 & 19.1 & 26.2 & 27.8 \\
\hline Chlorides, mg/L & $(10)$ & $(15)$ & $(25)$ & $(40)$ & $(60)$ & $(85)$ & $(115)$ & 150 & 177 & 201 & 291 \\
\hline
\end{tabular}

The frequency of the bounded daily dynamics $\mathrm{Ni}$ of the relative humidity, the minimal wind direction, and the wind speed (all have $~ 5.4$-day period) are synchronised with the frequencies of the base dynamics $\mathrm{Nb}$ of the Lake Ontario level (the same 5.4-day period) and air pressure (5.6-day period). This illustrates that these variables are bounded with the local source of water. When the relative humidity, the minimal wind direction, wind speed and air pressure come into resonance with the extremely high temperature drops at hourly scales, the perfect condition for the extreme convection has being created as the self-stabilization mechanism. Two variables, relative humidity and wind direction, are the key ones for self-stabilization, which is indicated by negative and equal PBD and PID in the hourly scale for both of them (Table 1). They both characterise extremely flexible energy and water storage capacity of an instant column of atmosphere, which depend on temperature, its gradient, and water available to fit 
it. If there is not enough water available in the atmosphere for the instant temperature gradient, the twister, in which the same water turns thousand times around per minute providing the avalanche-type condensation (Ivanov [19]), is the perfect solution for this extremely localized energy consumption and tension release technique.

Based on Table 2, yearly averages, there is the increasing trend in temperature and the light decreasing trend in relative humidity, indicating the increasing insufficiency of atmospheric moisture. At the same time, the increasing trends in all daily dynamics of relative humidity (dQb, dQi and dQmax) and the base dynamics of all wind variables indicate the dynamic feedback effect to compensate the lack of moisture. This is the right moment to remind that the function to return water to the atmosphere belongs to the naturally developed vegetation, which also keeps the cool temperature of its surface pumping 1000 times more water from the ground than it needs for its own biomass production (Bouten [7]). The maximal deforestation of the Humber watershed that was ever entirely forested had happened before the examined period, changing during the period from $10.3 \%$ to $18.2 \%$, PODPD [11] and TRCA [22]. A very noticeable shift in base and interflow and drop of the storm component in the late $70 \mathrm{~s}$ coincides with the beginning of storm water management practices which were introduced by the MOE in 1980, [12]. This slows down drainage as well as the process of washing out the urban contamination, particularly chlorides, and speeding up their accumulation. This explains why almost double-increased base dynamic of the maximal wind speed cannot provide the required rate of evaporation: there is no fast local access to this bounded by chlorides water.

The oscillation of the inter-storm components in the equilibrium point shows a steady stage (the highest sum of the base 0.38 and inter component) at all scales for the last 2002-2006 pentad. This means that the dynamic structures at all scales are in the same phase of oscillation (the resonance condition) and the upcoming years (pentads) may be characterised by the abrupt change to the most unstable dynamic composition in all scales initiating the abrupt climate change predicted by Gagosian in 2003 [18].

Based on the cross-correlation results it is possible to state that temperature is the factor which is controlled at different scales by different combinations of the water cycle variables because temperature components are always in opposition to any leading variable. However, total and base component of the river flow are the leading variables for the lump set in the yearly scale. Furthermore, baseflow opposes itself in the leading pare for components indicating the conflict between natural and anthropogenic development in long-term trends (the yearly scale).

\section{Conclusions}

Based on the results of 1953-2006 hydrological investigation of the Humber River watershed which included the HFA application, the following conclusions have been made: 
- The multi-scale dynamic structure of the hydrosphere within the specified spacetime reveals well pronounced trends toward the possible abrupt instability, which are not seen in the conventional averages of totals;

- The quantitative increase of the bounded water resources caused by continual urbanization and proper water management in combination with the increasing contamination at the Humber River result in alteration of the water cycle's functional profile, i.e. the drainage function takes over the temperature control reducing the atmospheric moisture;

- Tornado represents a self-stabilisation mechanism at the hourly scale under the insufficient for temperature gradients amount of the local atmospheric moisture;

- HFA is the reliable technical tool for sustainability assessment, which is defined as the functional stability of the hydrological cycle within a developing spacetime.

\section{Acknowledgements}

I would like to take this opportunity to express my deep appreciation to Ernst Zaltsberg, hydrogeologist, PhD., P. Geo., Julia Vedom, MA, and Elizabeth Bedwell for their editorial help during the article preparation; Dean Young, coordinator of watershed planning at TRCA, for help in obtaining archive information.

\section{References}

[1] Murtugudde, R., Regional Earth System prediction: a decision-making tool for sustainability? Current opinion in Environmental Sustainability, 1, pp. 37-45, 2009.

[2] Hoff, H., Global water resources and their management. Current opinion in Environmental Sustainability, 1, pp. 141-147, 2009.

[3] Soroko, E., Structural harmony of systems, Minsk: Nauka I Tekhnika, 1984 (in Russian)

[4] Stakhov, A.P., Mathematics of Harmony: From Euclid to Contemporary Mathematics and Computer Science, World Scientific Publishing Company, 600 p., 2009

[5] Ibanez, J.G., Hernandez-Esparza, M., Doria-Serrano, C., Fregoso-Infante, A., \& Singh, M.M., Environmental Chemistry. Fundamentals. Springer, New York, 334 p., 2007.

[6] Beran, M., The Role of Water in Global Environmental Change Processes. NATO ASI Series, Vol. 31, The Role of Water and Hydrological Cycle in Global Change, pp. 1-22, 1995.

[7] Bouten, W., Plant Control on Evapotranspiration: Models and Measurements. NATO ASI Series, Vol. 31, The Role of Water and Hydrological Cycle in Global Change, pp. 105-133, 1995. 
[8] Peixoto, J.P., The role of the atmosphere in the water cycle. NATO ASI Series, Vol. 31, The Role of Water and Hydrological Cycle in Global Change, pp. 199-252, 1995.

[9] Rowntree, p., \& Dumenil, L., Hydrology in Climate Models and Effects on Climate. NATO ASI Series, Vol. 31, The Role of Water and Hydrological Cycle in Global Change, pp. 59-104, 1995.

[10] Berthouex, P.M. \& Brown, L.C. Statistics for Environmental Engineers, Lewis Publishers, Second edition, 522p, 2002.

[11] Province of Ontario Department of Planning and Development (PODPD), Humber Valley Report, 606 p., 1948.

[12] Ministry of the Environment (MOE). Stormwater Management Planning and Design Manual. Ontario: MOE, ISBN 0-7794-2969-9, March 2003.

[13] Xie, T., Liu, X., \& Sun, T., The effects of groundwater table and flood irrigation strategies on soil water and salt dynamics and reed water use in the Yellow River Delta. Ecological Modelling, Volume 222, Issue 2, pp. 241-252, 2011.

[14] Vedom, R. Daily Chloride Contamination of Lake Ontario by Etobicoke Creek, The Sustainable City II, Urban Regeneration and Sustainability, Advances in Architecture, WIT press, pp. 631-641, 2002.

[15] Vedom, R. The SimpleBase delineation model: a new tool for water investigation and assessment, Proc. of the $12^{\text {th }}$ World Water Congress, November 22-25, New Delhi, 2005.

[16] Vedom, R., The hydrological limits of urbanization, Proc. of the $59^{\text {th }}$ Annual Conference of CWRA, Toronto, June 2-5, 2006.

[17] Doswell, C.A. \& Bosart, L.F., Extratropical synoptic-scale processes and severe convection. Meteorological Monograph: 2001. http://www.cimms.ou.edu/ doswell/Monograph/Synoptics.html

[18] Gagosian, R.B., Abrupt Climate Change: Should we be worried? 2003. http://www.whoi.edu/page.do?cid=9986\&pid=12455\&tid=282

[19] Ivanov, V.A., Tropical cyclones, tornado. 6p., 2004. http://psy.tom.ru/tornado.html

[20] Smith, S.W., The Scientist and Engineer's Guide to Digital Signal processing, 1997. www.dspguide.com

[21] Sontag, E. D., Mathematical Control Theory: Deterministic Finite Dimensional Systems. Second Edition, Springer, New York, 1998. http://www.math.rutgers.edu/ sontag/mct.html

[22] Toronto and Region Conservation Authority (TRCA), TRCA: Humber River State of the Watershed Report - Land and Resource Use, 2008. (http://trca.on.ca/protect/watersheds/humber-river/resources.dot)

[23] Vedom, R., The Structural Harmony Chart of Hydrosphere: the efficient element of the Separated Flux Analysis, 2007 (in Russian), (www.trinitas.ru/rus/doc/0232/004a/02320172.htm) 